\title{
TEST SITUACIONAL DESARROLLO DE LAS COMPETENCIAS BÁSICAS DE EMPLEABILIDAD: VALIDACIÓN DE UN INSTRUMENTO PSICOEDUCATIVO PARA LA INTERVENCIÓN SOCIOEDUCATIVA
}

\section{SITUATIONAL JUDGMENT TEST OF BASIC EMPLOYABILITY COMPETENCES DEVELOPMENT: VALIDATION OF A PSYCHOEDUCATIVE INSTRUMENT FOR SOCIOEDUCATIVE INTERVENTION}

\section{TESTE SITUACIONAL PARA O DESENVOLVIMENTO DE COMPETÊNCIAS BÁSICAS DE EMPREGABILIDADE: VALIDAÇÃO DE UM INSTRUMENTO PSICOEDUCACIONAL DE INTERVENÇÃO SOCIOEDUCACIONAL}

\author{
Josefina SALA-ROCA*, Mercè JARIOT-GARCIA*, Laura ARNAU-SABATÉS* \\ \& Eduardo DOVAL \\ *Universidad Autónoma de Barcelona
}

PALABRAS CLAVE: empleabilidad;

habilidades básicas de empleabilidad

habilidades clave de empleabilidad,

test situacional; pedagogía social
RESUMEN: Las competencias básicas de empleabilidad, también denominadas competencias clave, son fundamentales para acceder, mantener y promocionar en el empleo, y son especialmente relevantes en la promoción de la inserción laboral de los jóvenes desfavorecidos.

En este estudio se describe el desarrollo de un instrumento psicoeducativo copyleft basado en el modelo de Arnau et al. (2014) que permite a los profesionales de la pedagogía social evaluar las competencias básicas de empleabilidad en adolescentes y jóvenes de 12 a 18 años: El Test Situacional de Desarrollo de Competencias de Empleabilidad.

Se diseñaron 29 situaciones-problema que se sucedían a lo largo de 5 historias de la vida cotidiana de cualquier adolescente. Se entrevistó a 102 adolescentes y jóvenes preguntándoles cómo reaccionarían ante dichas situaciones-problema. El análisis de contenido de dichas respuestas permitió seleccionar las respuestas más frecuentes indicativas de diferentes niveles de desarrollo competencial. 10 expertos asignaron las situaciones-problema a una de las 8 competencias propuestas por Arnau et al. (2014) y posteriormente asignaron puntuaciones de entre $\mathrm{O}$ a 5 a las diferentes respuestas en función del nivel de desarrollo que indicaban las respuestas, con altos niveles de acuerdo.

CONTACTO CON LOS AUTORES

JOSEFINA SALA ROCA. Facultat de Ciències de l'Educació, despacho G6-171.

Universitat Autònoma de Barcelona. E-mail: Fina.Sala@uab.cat. 


\begin{tabular}{|c|c|}
\hline & $\begin{array}{l}\text { Después de una aplicación piloto a un grupo de } 47 \text { jóvenes, que permitió comprobar la } \\
\text { variabilidad de las respuestas, se administró el test a 1,011 jóvenes procedentes de } 6 \text { centros } \\
\text { formativos. Del análisis factorial de las respuestas emergió una estructura unifactorial de } 23 \\
\text { ítems, con valores elevados de ajuste del modelo y de la fiabilidad. } \\
\text { Los resultados obtenidos avalan la validez de contenido, ecológica y de constructo del } \\
\text { test DCBE recibiendo una buena aceptación tanto entre los alumnos como entre los profeso- } \\
\text { res por su atractivo y utilidad. El test posibilita iniciar la preparación para la empleabilidad en } \\
\text { etapas tempranas previas a la formación postobligatoria. Su carácter copyleft posibilita una } \\
\text { amplia utilización por parte de los profesionales de la Pedagogía Social. }\end{array}$ \\
\hline $\begin{array}{l}\text { KEY WORDS: } \\
\text { employability; } \\
\text { basic employability } \\
\quad \text { skills; } \\
\text { key employability } \\
\quad \text { skills; } \\
\text { situational test; } \\
\text { social pedagogy }\end{array}$ & $\begin{array}{l}\text { ABSTRACT: Basic employability competences, also known as key competences, are essential } \\
\text { in accessing, retaining and advancing in employment, and are especially relevant in promoting } \\
\text { the employment of disadvantaged young people. } \\
\text { This study describes the development of a copyleft psychoeducational instrument based } \\
\text { on the model posited by Arnau et al. (2014) that Social Pedagogy professionals can use to } \\
\text { evaluate the basic employability competences in adolescents and young people aged } 12 \text { to } 18 \text { : } \\
\text { The Situational Test of Basic Employability Competences Development (DCBE). } \\
\text { A total of } 29 \text { problem situations were designed and included within five stories related to } \\
\text { the daily life of any adolescent. A total of } 102 \text { adolescents and young people were interviewed } \\
\text { and asked how they would react to these problem situations. The responses were subjected } \\
\text { to a content analysis in order to select the most frequent responses indicative of different } \\
\text { levels of competence development. Ten experts then assigned the problem situations to one } \\
\text { of the eight competences proposed by Arnau et al. (2O14), before assigning scores of o to } 5 \text { to } \\
\text { the different responses based on the level of development indicated by the responses, with } \\
\text { high levels of agreement. } \\
\text { Following a pilot study on a group of } 47 \text { young people to verify the variability of the } \\
\text { responses, the test was administered to } 1,011 \text { young people at six training centres. A factor } \\
\text { analysis of the responses yielded a unifactorial structure of } 23 \text { items, with high values for } \\
\text { model fit and reliability. } \\
\text { The results obtained support the content, ecological and construct validity of the DCBE } \\
\text { test, which was well accepted by both students and teachers for its attractiveness and use- } \\
\text { fulness. The test makes it possible to begin preparing students for employment in the early } \\
\text { stages prior to post-compulsory training. Its copyleft nature means it can be widely used by } \\
\text { Social Pedagogy professionals. }\end{array}$ \\
\hline $\begin{array}{l}\text { PALAVRAS-CHAVE: } \\
\text { empregabilidade; } \\
\text { habilidades } \\
\text { básicas de } \\
\text { empregabilidade; } \\
\text { principais } \\
\text { habilidades de } \\
\text { empregabilidade; } \\
\text { teste situacional; } \\
\text { pedagogia social }\end{array}$ & $\begin{array}{l}\text { RESUMO: As competências básicas de empregabilidade, também chamadas de competên- } \\
\text { cias-chave, são essenciais para o acesso, manutenção e promoção do emprego, sendo espe- } \\
\text { cialmente relevantes para a promoção da inserção laboral de jovens desfavorecidos. Este } \\
\text { estudo descreve o desenvolvimento de um instrumento psicoeducacional copyleft baseado } \\
\text { no modelo de Arnau et al. (2O14) que permite aos profissionais da pedagogia social avaliar as } \\
\text { competências básicas de empregabilidade em adolescentes e jovens de } 12 \text { a } 18 \text { anos: Teste } \\
\text { Situacional para o Desenvolvimento de Competências de Empregabilidade (DCBE). } \\
\text { Foram projetadas } 29 \text { situações-problema que ocorreram ao longo de } 5 \text { histórias da vida } \\
\text { diária de qualquer adolescente. } 102 \text { adolescentes e jovens foram entrevistados e questiona- } \\
\text { dos sobre como reagiriam a essas situaçães problemáticas. A análise de conteúdo dessas } \\
\text { respostas permitiu selecionar as respostas mais frequentes indicativas de diferentes níveis de } \\
\text { desenvolvimento de competências. } 10 \text { especialistas atribuíram as situações-problema a uma } \\
\text { das } 8 \text { competências propostas por Arnau et al. (2014) e posteriormente atribuíram notas de o } \\
\text { a } 5 \text { às diferentes respostas com base no nível de desenvolvimento indicado pelas respostas, } \\
\text { com altos níveis de concordância. } \\
\text { Após uma aplicação piloto a um grupo de } 47 \text { jovens, que permitiu verificar a variabilidade } \\
\text { das respostas, o teste foi aplicado a } 1,011 \text { jovens de } 6 \text { centros de formação. Da análise fatorial } \\
\text { das respostas, emergiu uma estrutura unifatorial de } 23 \text { itens, com altos valores de ajuste do } \\
\text { modelo e confiabilidade. } \\
\text { Os resultados obtidos suportam a validade de conteúdo, ecológica e de construto do } \\
\text { teste DCBE, obtendo boa aceitação tanto por parte dos alunos como dos professores pela } \\
\text { sua atratividade e utilidade. O teste permite iniciar a preparação para a empregabilidade } \\
\text { nas fases iniciais, antes da formação pós-obrigatória. Seu caráter copyleft permite sua ampla } \\
\text { utilizacão por profissionais da Pedagogia Social. }\end{array}$ \\
\hline
\end{tabular}




\section{Introducción}

\subsection{La Empleabilidad como Estrategia para Afrontar el Paro Juvenil}

Una de las principales dificultades que tienen que afrontar los países occidentales es la alta tasa de paro. El trabajo se ha convertido en un bien escaso, además las posibilidades de acceso no son las mismas para todos (Olea, 2001). A ello se debe sumar los bajos salarios que sumergen a muchos hogares en la pobreza. Después de la crisis en la Eurozona, la desigualdad continúa creciendo a pesar de que globalmente las economías se hayan recuperado (Dreger et al., 2015).

La tasa de paro en la Eurozona alcanzaba, en diciembre de 2019, el $7.3 \%$ de la población general, siendo España uno de los países con índices más altos de paro (14.7\%), solo superada por Grecia (17.3\%). No obstante, los índices de paro juvenil doblan los de la población general. En la misma fecha, el $15.4 \%$ de los jóvenes menores de 25 años estaba en paro, cifra que entre los jóvenes españoles se elevaba al $30.5 \%$. En 2020 estos datos han empeorado ubicándose en el $18.1 \%$ y $40.45 \%$ a raíz de la crisis de la covid19 (Eurostat, 2020).

La tasa de paro no se distribuye de manera homogénea entre la población juvenil ya que afecta sobretodo a los menores de edad, a los jóvenes menos formados, a los pertenecientes a contextos socioeconómicos humildes y a jóvenes de otras nacionalidades (Moreno, 2015).

Es por ello que la empleabilidad es un elemento central en las políticas de empleo en la Unión Europea (Crisp \& Powell, 2016; McQuaid, \& Lindsay, 2005). La Empleabilidad es un concepto que gira en torno a cualidades y competencias requeridas en una persona para ajustarse a las demandas de oferta laboral y progresar en el mercado de trabajo (Bridgstock, 2009; Lo Presti, \& Pluviano, 2016). Las competencias o habilidades de empleabilidad aportan sentido a la capacidad individual a largo plazo para desarrollar una carrera profesional y progresar en el mercado laboral (Curtis \& McKenzie, 2001). En este sentido Breen (2005) señala que los sistemas educativos focalizados en las competencias requeridas para el empleo se asocian a bajas tasas de paro juvenil.

\subsection{Empleabilidad y Competencias de Empleabilidad}

El concepto de empleabilidad se ha desarrollado para describir los objetivos de las estrategias económicas de las grandes instituciones a nivel nacional, regional y local (McQuaid \& Lindsay, 2005). Esto ha conllevado numerosas iniciativas orientadas a dotar de una cualificación profesional a los jóvenes que quieren insertarse en el mundo laboral y a las personas desempleadas, y a asociar la cualificación profesional al éxito en el acceso al mundo laboral (McQuaid, 2006; Organización Internacional del Trabajo, 2014). Esta visión conlleva la propuesta de modelos de competencias específicas asociadas a la profesión o a los puestos de trabajo.

Sin embargo, como señalan Nickson et al., (2012), disponer de calificación profesional no es el único factor explicativo del acceso al mundo laboral. Hay competencias transversales que también son necesarias para acceder al empleo, tanto si este requiere de una baja, como de una alta cualificación profesional. En esta línea se han desarrollado propuestas que emplean diversas terminologías referidas a un mismo concepto asociado a habilidades o competencias de empleabilidad; algunas veces denominadas como "soft skills", habilidades genéricas, nucleares, básicas o habilidades clave de empleabilidad (Curtis \& McKenzie, 2001). Estas competencias permiten a los trabajadores cualificados, y también a los no cualificados, participar en variados puestos de trabajo (Nisha \& Rajasekaran, 2018). En este artículo adoptamos la definición de competencias básicas de empleabilidad (Arnau et al., 2014) que las definen como competencias básicas necesarias para acceder y mantener un puesto de trabajo independientemente del nivel de cualificación profesional.

Frente a la necesidad de que la educación promueva el desarrollo de las habilidades clave para la empleabilidad, ha surgido un nuevo concepto, las habilidades de empleabilidad transferibles. Estas son habilidades que se desarrollarían desde la educación y serían transferibles al entorno laboral (Fallows \& Steven, 2000, citado por Muhamad, 2012). Como señala Muhamad (2012), los empleadores no solo reclutarían a los graduados por sus conocimientos y habilidades técnicas que pueden comprobar en su expediente académico, sino por las habilidades transferibles y atributos personales que van más allá de las habilidades técnicas y que les permiten afrontar los requerimientos de los puestos de trabajo de forma exitosa.

Diversos autores han desarrollado modelos de competencias clave o genéricas que serían necesarias para diversos puestos de trabajo (ver una revisión en Lo Presti \& Pluviano, 2016). Otros centran el modelo en las competencias básicas para el desarrollo de la propia carrera profesional (Bridgstock, 2009; Lo Presti, 2009); y también se han desarrollado modelos holísticos que intentan abarcar un amplio espectro de competencias y/o habilidades que puedan explicar el éxito en la 
incorporación al mundo laboral (McQuaid \& Lindsay, 2005; Olmos-Rueda \& Mas-Torelló, 2017).

Sin embargo, no existen modelos que propongan competencias básicas de empleabilidad necesarias para cualquier puesto de trabajo, independientemente del sector económico o la cualificación profesional (Arnau et al. 2014). La mayoría de los modelos anteriormente citados se plantean desde la perspectiva de la formación postobligatoria, y habitualmente incluyen conocimientos académicos, aunque sean básicos. No obstante, muchos jóvenes, como aquellos de entornos desfavorecidos (procedentes de familias con escasa capacidad formativa y pocos recursos económicos, centros de tutela, etc.) que son atendidos desde diversos programas sociales, acaban ocupando puestos no cualificados que, si bien no precisan de altas habilidades técnicas, sí requieren competencias básicas de empleabilidad. Muchos de estos jóvenes no logran mantener estos puestos de trabajo por la falta de competencias como el sentido de la responsabilidad para llegar a tiempo al trabajo, la resolución de problemas, no saber trabajar en equipo, etc. (Olmos-Rueda \& Mas-Torelló, 2017; Alonso et al., 2018).

Es por ello que Arnau et al. (2014), después de analizar el marco conceptual de la empleabilidad y a partir de la evaluación de especialistas, proponen un modelo que incluye 8 competencias básicas de empleabilidad necesarias para adquirir y mantener un puesto de trabajo, independientemente del tipo de trabajo y del sector profesional y que pueden ser entrenables a edades tempranas. Estas competencias son: responsabilidad y co-responsabilidad, trabajo en equipo, comunicación, perseverancia, flexibilidad, autoorganización, construcción del proyecto educativo-profesional y toma de decisiones y resolución de problemas. Estas competencias se adquieren durante la segunda infancia y la adolescencia, principalmente a través de la educación familiar y escolar, son transferibles a los puestos de trabajo y están en constante evolución a lo largo de la vida.

\subsection{Los Test Situacionales y la Medición de las Competencias de Empleabilidad}

La medición de las habilidades laborales para facilitar los procesos de selección de personal es uno de los principales focos de interés de la psicología del trabajo. Se han propuesto diferentes técnicas para medir diversas habilidades con la finalidad de seleccionar a los trabajadores idóneos para puestos de trabajo específicos, como son los test de inteligencia, de personalidad, entrevistas y cuestionarios ad hoc (Lolito, 2015). Esta disparidad de instrumentos también se observa en las tentativas de medición de las competencias de empleabilidad. Así se han utilizado cuestionarios de autoevaluación (Rothwell, et al. 2008; Griffiths et al. 2018), checklist (Blalock et al. 2006), rúbricas (Riebe \& Jackson, 2014), cuestionarios de evaluación de los empleadores (Abas \& Imam, 2016), etc. No obstante, la mayoría de estas pruebas se centran en las etapas prelaborales y etapas universitarias e incluyen el dominio de habilidades académicas que pueden no ser necesarias para empleos básicos.

En la última década, los test situacionales han irrumpido en el ámbito de selección de personal logrando una gran aceptación porque permiten medir habilidades de tipo interpersonal y habilidades específicas del lugar de trabajo a grandes grupos en las primeras fases de dicha selección con una mayor validez predictiva (Lievens et al., 2008). En estos test se presentan diferentes situaciones-problema específicas relacionadas con el puesto de trabajo y se pregunta por la probabilidad con que se realizaría esa acción o por la efectividad de ella. Algunas de las fortalezas de los test situacionales es que suelen obtener mejores evidencias de validez de criterio que test tradicionales (Lievens et al., 2008), son más atractivos que los test convencionales y presentan menos sesgos hacia las minorías. Sin embargo, al ser específicos del contexto son más laboriosos de elaborar, ya que deben ser desarrollados para puestos de trabajo concretos o culturas específicas (Sorrel et al., 2016).

El desarrollo de un test situacional (Lievens et al., 2008) requiere que en una primera fase se seleccionen tanto los incidentes críticos o situaciones laborales representativos de las competencias que se desea evaluar, como los escenarios donde estas se desarrollaran. En una segunda fase, un grupo de empleados con experiencia y otro con poca experiencia generan posibles respuestas a cada situación, obteniéndose así respuestas expertas e inexpertas. Los desarrolladores del test editan dichas respuestas a las que, en una fase final, un grupo de expertos identificaran y asignaran puntuaciones.

\section{Justificación y objetivos}

No hemos encontrado en la literatura consultada ningún test situacional para medir competencias básicas de empleabilidad dirigidas a las primeras etapas de la adolescencia. Cuando los adolescentes llegan a la edad laboral, con o sin estudios profesionalizadores, ya suelen disponer de habilidades básicas de empleabilidad cuyo nivel de desarrollo será clave para el acceso, mantenimiento y promoción en el puesto trabajo. Competencias 
como la responsabilidad, el trabajo en equipo, la comunicación, la perseverancia, la flexibilidad, la toma de decisiones y resolución de problemas, la autoorganización, y la construcción del proyecto educativo-profesional propuestas en el modelo de Arnau et al. (2014) se desarrollan inicialmente en el contexto familiar y escolar gracias al acompañamiento de los educadores, padres y madres, y se consolidan como competencias transferibles al empleo. Cimatti (2016) también coincide en que estas competencias se adquieren a lo largo del desarrollo, y señala que no son responsabilidad única de universidades y empresas, sino que deberían enseñarse en la educación primaria. Disponer de un test situacional que evalúe el nivel de desarrollo de dichas competencias constituiría un valioso instrumento de diagnóstico educativo a partir del cual diseñar planes individualizados, y ayudaría a los jóvenes a desarrollar estas competencias clave de empleabilidad para favorecer su futura inserción laboral y progreso profesional.

El objetivo de este trabajo es validar el Test Situacional de Desarrollo de Competencias de Empleabilidad (Sala et al. 2020), un instrumento copyleft dirigido a adolescentes y jóvenes de entre 12 y 18 años. Este instrumento tiene un especial interés en el ámbito de la Pedagogía Social para promover procesos formativos orientados a la inserción sociolaboral de aquellos colectivos de jóvenes más vulnerables, si bien tienen validez para cualquier adolescente de estas edades.

\section{Metodología}

El Test Situacional de Desarrollo de Competencias Básicas de Empleabilidad (DCBE) (Sala et al. 2020), fundamentado en el Modelo de Competencias Básicas de Empleabilidad IARS (Arnau et al. 2014), se ha creado considerando las 8 competencias básicas necesarias para adquirir y mantener cualquier puesto de trabajo. Se siguió el procedimiento descrito por Lievens et al., (2008) para la creación de test situacionales y se comprobó su estructura interna y fiabilidad como se describe en la figura 1.

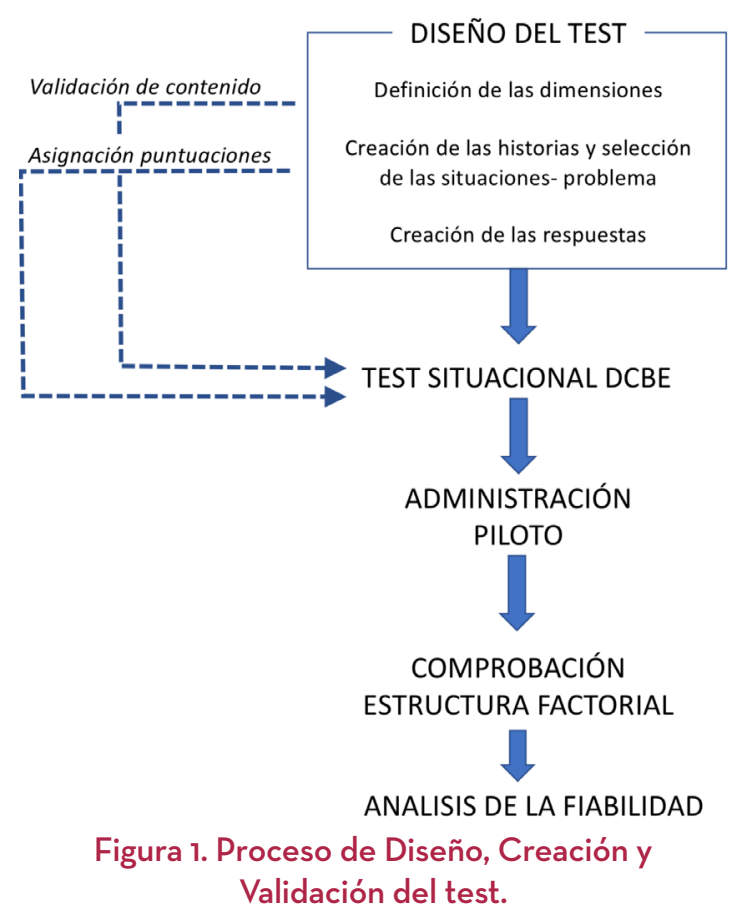

En una primera etapa se desarrolló el test diseñando un conjunto de situaciones-problema y entrevistando a 102 adolescentes para definir las opciones de respuesta. Posteriormente, un grupo de especialistas realizó una primera validación del test y asignó las puntuaciones a las opciones de respuesta. En una primera administración piloto a una muestra de 47 adolescentes y jóvenes de entre 12 y 18 años se valoró la variabilidad de las respuestas y otros aspectos asociados al proceso de respuesta como el nivel de comprensión, el tiempo de respuesta, el nivel de cansancio, etc. Finalmente, se aplicó el instrumento a 1011 jóvenes de las mismas edades con el objeto de analizar la estructura factorial y la fiabilidad de sus puntuaciones. Estos jóvenes procedían de los institutos de la ciudad de Mollet con la que se realizó un acuerdo de colaboración, en la que se incluía todo el espectro de jóvenes, tanto si procedían de entornos normalizados como vulnerables. Si bien el estudio perseguía desarrollar un instrumento copyleft útil para los profesionales de la Pedagogía Social que trabajan con jóvenes desfavorecidos, la validación debía realizarse con todo el espectro de población para poder contar con baremos representativos. 


\section{Diseño, desarrollo y validación del test DCBE}

\subsection{Fase preliminar: Identificación de Antecedentes y Definición de Dimensiones (variables) e Indicadores}

Para desarrollar el test se ha utilizado el Modelo de Competencias Básicas de Empleabilidad de Arnau et al. (2014) que plantea 8 competencias necesarias para adquirir y mantener un puesto de trabajo, independientemente del tipo de trabajo: Autoorganización, Construcción del proyecto profesional, Toma de decisiones y resolución de problemas, Trabajo en equipo, Comunicación, Perseverancia, Flexibilidad, Responsabilidad y Co-responsabilidad. Estas competencias fueron validadas por expertos en el ámbito (Arnau et al., 2014), y por una amplia y diversa muestra de trabajadores de los diferentes sectores económicos (Jariot et al. 2021).

\subsection{Fase 1: Creación de las Historias y Selección de las Situaciones-Problema}

Como ya hemos señalado, el nivel de logro de una competencia puede variar en función del contexto, y por este motivo se contemplaron diferentes ambientes de socialización de los adolescentes y jóvenes de 12 a 18 años, como son el instituto, la familia, los amigos y el ocio. Teniendo en cuenta estos contextos se diseñaron 5 historias (ver Tabla 1) en las que se suceden varias situaciones-problema. Aunque no todos los jóvenes hayan vivido estas situaciones-problema, sí habrán experimentado situaciones-problema similares y es de suponer que la proyección a estas situaciones-problema no suponga ninguna dificultad.

Tabla 1. Historias y contextos en que se desarrollan

\begin{tabular}{|c|c|c|}
\hline Contexto & Historia & $\begin{array}{c}\text { Número de } \\
\text { situaciones-problema }\end{array}$ \\
\hline Escolar & $\begin{array}{c}\text { Tengo muchos } \\
\text { deberes }\end{array}$ & 5 \\
\hline Familiar & La mascota & 6 \\
\hline Amistades & La fiesta & 7 \\
\hline Ocio & El tenderete & 6 \\
\hline Escolar & Eres delegado & 5 \\
\hline
\end{tabular}

El equipo de investigación, formado por expertos en el ámbito de empleabilidad, diseñó entre 5 y 7 situaciones-problema para cada historia, ante las que un adolescente o joven puede reaccionar de diferente manera en función de su nivel competencial. En total se plantearon 29 situaciones que requerían de las ocho competencias propuestas en el Modelo de Competencias Básicas de Empleabilidad (Arnau et al., 2014).

Al definir y describir los personajes de las diferentes historias se contempló la perspectiva de género alternando al azar personajes femeninos y masculinos para evitar las respuestas bajo estereotipos sociales.

Otro elemento que se consideró es el hecho que no todos los adolescentes y jóvenes tienen una familia tradicional, algunos pueden estar a cargo de familias de acogida y otros pueden estar residiendo en centros de protección, por lo que no conviven con sus figuras parentales, $u$ otros modelos familiares. Es por ello que en la revisión del contenido del test se eliminaron todas las referencias a las figuras parentales y de hermanos. En el caso de que fuera necesario referirse a una figura funcional parental, se optó por el término genérico "adultos".

\subsection{Fase 2: Creación de las Respuestas}

Con el fin de que cada situación-problema tuviera un listado de respuestas representativas de las conductas de los jóvenes, se aplicó una entrevista semiestructurada a 102 adolescentes y jóvenes ( 57 chicas y 34 chicos) en la que se planteaban las distintas historias y situaciones-problema y se indagaba por cómo se comportarían en cada una de ellas. El número de adolescentes y jóvenes entrevistados, por franjas de edad fue el siguiente: 12-13 años ( $n=29), 14-15$ años $(n=29), 16-17$ años $(n=29)$ y 17-19 años $(n=15)$.

Se informó a los participantes del propósito de la entrevista, así como de que la información seria tratada confidencialmente y se les pidió su consentimiento verbal. Todas las respuestas fueron registradas y posteriormente transcritas. Con ellas se realizó un análisis de contenido categorizándolas en las distintas competencias de empleabilidad.

El equipo de investigación seleccionó, de entre las reacciones más frecuentes, cinco opciones de respuestas para cada situación indicativas de distintos niveles competenciales.

\subsection{Fase 3: Valoración de la Adecuación del Contenido de las Historias por Expertos}

Con el fin de asignar las diferentes situaciones-problema a las competencias que se desean representar, se seleccionó un panel de 10 
expertos del ámbito de la formación laboral (5) y selección de recursos humanos (5). Se solicitó al grupo de expertos qué competencias podían valorarse con cada situación-problema del test. Para ello se elaboró un cuestionario que incluía las 5 historias con las situaciones-problema y opciones de respuesta. Los jueces debían valorar si la situación-problema permitía medir la competencia (1) O no permitía medirla (o).

Para cada situación-problema valorada se calculó la mediana de las puntuaciones que cada juez había asignado a cada una de las competencias (ver Tabla 2). Para cada experto también se calculó su diferencia en valor absoluto respecto a la mediana de puntuaciones del conjunto de jueces. Dos de los jueces (6 y 7) superaron una discrepancia total del conjunto de situaciones de 9 puntos, que se consideró excesiva, por lo que las valoraciones de estos jueces no se consideraron en el análisis posterior (ver Tabla 2).

Tabla 2. Discrepancia, en términos absolutos, de la valoración de cada juez respecto a la mediana de valoraciones

\begin{tabular}{|l|c|c|c|c|c|c|c|c|c|c|}
\hline \multicolumn{1}{|c|}{ Historia } & Juez 1 & Juez 2 & Juez 3 & Juez 4 & Juez 5 & Juez 6 & Juez 7 & Juez 8 & Juez 9 & Juez 10 \\
\hline H1 deberes & 2,1 & 0,4 & 1,6 & 1,1 & 0,8 & 1,8 & 2,4 & 0,8 & 0,9 & 1,3 \\
\hline H2 mascota & 1,7 & 1,7 & 1,3 & 1,2 & 1,0 & 3,7 & 0,8 & 0,5 & 1,0 & 1,3 \\
\hline H3 fiesta & 1,1 & 1,0 & 1,3 & 1,9 & 2,0 & 2,4 & 2,7 & 0,6 & 0,9 & 0,9 \\
\hline H4 tenderete & 2,4 & 0,8 & 1,4 & 2,8 & 1,3 & 2,8 & 3,4 & 0,8 & 0,8 & 1,9 \\
\hline H5 delegado & 1,4 & 1,7 & 1,5 & 0,9 & 1,5 & 2,9 & 2,9 & 2,1 & 1,3 & 1,3 \\
\hline Sumatorio & 8,7 & 5,5 & 7,1 & 7,7 & 6,5 & 13,5 & 12,2 & 4,6 & 4,8 & 6,6 \\
\hline
\end{tabular}

Una vez eliminadas las valoraciones extremas de estos dos jueces, los niveles de acuerdo entre los jueces en cada situación, calculados con el índice kappa, oscilaron entre .85 y .91. De acuerdo con Fleiss (1981), los valores superiores a .75 se consideraron indicadores de un nivel alto de acuerdo. Solo una de las seis situaciones de la primera historia (Deberes) no alcanzó dicho grado de acuerdo entre jueces por lo que fue eliminada de la prueba. Las situaciones quedaron asignadas a las competencias en la que los jueces habían coincidido con valores de acuerdo superiores a .75. En la Tabla 3 puede observarse la asignación de cada situación-problema a las diferentes competencias resultante de la valoración de los expertos.

\begin{tabular}{|l|l|c|c|c|c|}
\hline \multicolumn{5}{|c|}{ Tabla 3. Situaciones-problema que permiten medir el nivel de cada competencia y } \\
puntuaciones asignadas por los expertos
\end{tabular}




\begin{tabular}{|c|c|c|c|c|c|}
\hline & $\begin{array}{l}\text { Historia: Tengo } \\
\text { muchos deberes }\end{array}$ & $\begin{array}{c}\text { Historia: } \\
\text { La mascota }\end{array}$ & $\begin{array}{l}\text { Historia: } \\
\text { La fiesta }\end{array}$ & $\begin{array}{c}\text { Historia: } \\
\text { El tenderete }\end{array}$ & $\begin{array}{c}\text { Historia: } \\
\text { Eres delegado }\end{array}$ \\
\hline Comunicación & D4 $\left(\begin{array}{llll}5 & \circ & 2 & 2\end{array}\right)$ & & $\mathrm{F}_{1}(1 \circ 444)$ & T6 (O $5 \circ 2$ 3) & $\mathrm{Del}_{4}(\mathrm{O} 5 \mathrm{5} 4 \mathrm{O}$ ) \\
\hline Flexibilidad & & & $\mathrm{F}_{4}\left(\begin{array}{lllll}5 & 3 & 3 & 2 & 0\end{array}\right)$ & $\begin{array}{l}\text { T3 (o } 35444) \\
\text { T5 (1 } 5455 \text { O) }\end{array}$ & Del3 (4 3224 5) \\
\hline Perseverancia & $\mathrm{D} 2(4 \circ 2 \circ 5)$ & 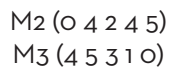 & F5 (1 352 O) & $\mathrm{T}_{4}(51230)$ & \\
\hline $\begin{array}{l}\text { Responsabilidad y } \\
\text { Co-responsabilidad }\end{array}$ & & M5 (4 1550$)$ & F7 (4 (4 $\left.\begin{array}{llll}5 & 2 & 2 & 1\end{array}\right)$ & & Del1 (1 $2 \circ 4$ 5) \\
\hline
\end{tabular}

NOTA. D1-5: ítems 1-5 de la historia “Tengo muchos deberes"; M1-6: La mascota; F1-7: La fiesta; T1-5: El tenderete; Del 1-5: Eres delegado. En los paréntesis se indican los valores asociados a cada una de las cinco opciones de respuestas siguiendo el orden de presentación.

\subsection{Fase 4: Baremación de las Respuestas}

Para asignar puntuaciones a las diferentes opciones de respuesta se siguió el procedimiento propuesto por McCann (2008). Se solicitó a 6 personas expertas ( 3 mujeres y 3 hombres, 3 del ámbito académico y 3 del ámbito de la orientación profesional) que asignaran una puntuación entre 0 y 5 a cada una de las opciones de respuesta: $\bigcirc$, cuando la respuesta señalaba ausencia de competencia, y 5 cuando la respuesta señalaba alto nivel competencial. Se calculó el grado de concordancia entre expertos a partir de las distancias de cada valoración respecto de la mediana de las puntuaciones asignadas a cada opción de respuesta. La concordancia entre las personas expertas fue alta, con un índice kappa de .9. Ninguna de ellas superó en promedio un punto de distancia a la mediana del grupo, por lo que no se descartaron las valoraciones hechas por ningún experto.

Para analizar la posible incidencia de la perspectiva de edad se solicitó a cinco estudiantes de los últimos cursos del grado de Pedagogía con formación en orientación profesional que también ponderaran las opciones de respuesta. Se analizó la concordancia entre las valoraciones realizadas por los estudiantes, siendo esta de .8. Ningún estudiante superó en promedio un punto de distancia de la mediana, por lo que tampoco en este caso se descartó ningún juez.

Las puntuaciones entre especialistas consolidados y en formación fue similar, siendo las diferencias en promedio de medio punto. Las medianas que se utilizaron para la baremación fueron las resultantes del conjunto de los expertos (consolidados y en formación) (ver Tabla 3).

\subsection{Fase 5. Primer Estudio Piloto para la Validación de la Prueba}

Se realizó un estudio piloto con 47 jóvenes (48.9\% chicos y $51.1 \%$ chicas) con edades comprendidas entre los 12 y los 18 años $(M=35, S D=2.1)$, contactados a partir de familiares y conocidos de los colaboradores del grupo de investigación. El objetivo de esta administración era comprobar la comprensión de todos los elementos de la prueba, el tiempo requerido para contestarla, el nivel de cansancio de las personas respondientes y la variabilidad de las respuestas dadas a las diferentes situaciones. Al final de la administración del test se preguntó a los participantes acerca de su experiencia con él.

No hubo problemas con la comprensión de las preguntas del test. El tiempo de respuesta osciló entre los 25 y 45 minutos. Todos los participantes coincidieron en valorar el test como muy ameno e interesante. En 23 de los 29 ítems del test, se seleccionaron todas las opciones de respuesta. Solo en 6 de los ítems una de las respuestas no fue seleccionada por ningún participante. Con todo ello se valoró que las opciones contempladas permitían recoger una amplia variabilidad de respuestas.

\subsection{Fase 6. Segundo Estudio Piloto para la Validación de la Prueba}

En este segundo estudio, se analizó la estructura interna de las situaciones de la prueba mediante un análisis factorial exploratorio, y se evaluó la fiabilidad de sus puntuaciones.

Sorrel et al. (2016) advierten sobre las dificultades de visualizar una estructura factorial en los test situacionales debido a la no independencia de las escalas y a la multidimensionalidad de los ítems. Considerando todas estas reservas, se procedió a realizar un análisis exploratorio de la estructura factorial para examinar si de este procedimiento emergía alguna solución factorial coherente con el modelo teórico de 8 dimensiones.

Se administró la prueba a una muestra de 1,011 jóvenes ( $48.5 \%$ chicos y $51.5 \%$ chicas), con edades comprendidas entre 12 y 18 años $(M=14.2, S D=1.6)$ 
procedentes de 6 centros formativos (cinco institutos y un centro de ocupación profesional) de la ciudad de Mollet de la provincia de Barcelona. El Instituto Municipal de Educación de Mollet del Vallés vinculado al Consistorio Municipal, contactó y convocó a la totalidad de los centros educativos del municipio que impartían educación secundaria (8 en total) a una reunión de presentación del proyecto y de la prueba a aplicar. Cinco de los ocho centros accedieron a participar de manera voluntaria. Los centros que se descartaron, reconocieron el interés de la prueba, y aludieron motivos externos al estudio. Además de los 5 centros educativos participantes, se sumó la participación del centro municipal de formación y ocupación que lleva a cabo acciones de orientación, formación y ocupación profesional.

Se realizó una sesión formativa con los profesores participantes para que todos aplicaran el mismo protocolo de administración. Los profesores administraron el test a sus alumnos y estos respondieron de forma anónima. Los alumnos ponían un código que solo ellos y sus profesores conocían. En reciprocidad se hizo una devolución de los resultados individuales a los jóvenes y a sus familias. Conjuntamente con los resultados se ponía a disposición de las familias y los centros una guía de actividades para desarrollar las competencias básicas de empleabilidad para familias y escuelas. Posteriormente a la administración del test se realizó un focus group con los profesores de cada centro para analizar la reacción de los alumnos ante el test, los tiempos de administración y si habían existido dificultades de comprensión.

Los centros informaron que prácticamente no habían existido problemas de comprensión en el test, los tiempos de respuesta oscilaron entre 3045 minutos, los alumnos habían mostrado interés por los resultados y habían encontrado el test entretenido. En muchos casos los alumnos se veían reflejados en las puntuaciones del test, y la opinión sobre si mismos coincidía con los resultados obtenidos. En general los centros también reconocieron el interés y la utilidad de la prueba. En tres de los seis casos, la prueba se utilizó para facilitar una orientación con el alumnado sobre las competencias a reforzar y una reflexión sobre sus características personales, habilidades, etc. Dos centros destacaron que las competencias que se trabajaban de manera transversal en el proyecto educativo de centro (en un caso el trabajo en equipo y, en el otro, la comunicación) se correspondían con las puntuaciones del test, que fueron en general más elevadas en dichas competencias.

Se utilizó en programa FACTOR 10.3. (Lorenzo-Seva \& Ferrando, 2007, 2011) para realizar un análisis factorial exploratorio que proporcionase evidencias de estructura interna de las puntuaciones del test. El análisis descriptivo inicial de los ítems señaló que tres de ellos (M2, M5, P5) presentaban distribuciones asimétricas (valores absolutos superiores a 1.96), y seis (M2, M3, M5, P1, P3 y $\mathrm{P} 5$ ) presentaban distribuciones apuntadas con valores de curtosis superiores a 1.96

Los valores de $\mathrm{KMO}=.816$ y de la prueba de esfericidad de Barlett $\left(\chi^{2}=4345.6\right.$; $d f=406, p<$ .0005) indican que los datos se adecúan a la realización de un análisis factorial.

El análisis paralelo sugirió una estructura de 2 factores, y dada la probable relación entre factores, se exploró esta opción con rotaciones oblicuas (Modelo 1). El modelo explicó un $27.4 \%$ de la variancia y presentó buenos índices de ajuste (ver Tabla 4); sin embargo, la distribución de los pesos factoriales no aportó una interpretación satisfactoria puesto que en uno de los factores solo pesaban dos de los cuatro ítems de la competencia "construcción del proyecto formativo profesional" y en el otro factor tenían un peso relevante la gran mayoría de los otros ítems. Después de analizar el contenido de esos ítems se decidió, prescindir de los 4 ítems de construcción del proyecto formativo profesional por ser muy diferentes al resto de los ítems.

\begin{tabular}{|l|c|c|c|c|c|c|c|}
\hline \multicolumn{7}{|c|}{ Tabla 4. Modelos factoriales del DCSE-J } \\
\hline Modelo & $\mathrm{n}$ & $\chi^{2}$ & $\mathrm{df}$ & $\mathrm{CFI}$ & TLI & GFI & RMSEA \\
\hline Modelo 1 & 1002 & $839.265^{* * *}$ & 349 & .97 & .97 & .96 & .026 \\
\hline Modelo 2 & 1002 & $498.808^{* * *}$ & 251 & .98 & .98 & .97 & .020 \\
\hline Modelo 3 & 1002 & $327.377^{* * *}$ & 230 & .97 & .96 & .98 & .024 \\
\hline NOTA. ${ }^{*} p<.05 ;{ }^{* *} p<.01 ; * * * .001$. \\
\hline
\end{tabular}

Una vez eliminados estos ítems se volvió a realizar un análisis factorial con adecuadas condiciones de aplicación (KMO = .838; Barlett $\left.\chi^{2}=3178 ; d f=300, p<.0005\right)$. El análisis paralelo 
sugirió de nuevo una solución de 2 factores (Modelo 2) que explicaba un $31.7 \%$ de la variancia. Ambos factores mostraron una elevada relación (.70). Sin embargo, la solución tampoco era interpretable.

Es por ello que se optó por analizar una solución unifactorial eliminando dos ítems que tenían pesos muy bajos en el modelo 2 (Modelo 3). En esta solución se obtuvieron unos índices satisfactorios de $\mathrm{KMO}=.851$, y Barlett $\chi^{2}=2951 ; \mathrm{df}=253, p$ $<.0005$. La solución resultante quedó constituida por 23 ítems, de los cuales 20 tenían cargas factoriales por encima de .30 y tres tenían valores entre .25 y .29. Esta solución explicaba el $25.2 \%$ de la variancia y unos valores de ajuste adecuados (Tabla 4) y su consistencia interna era buena (alfa = .75). En la Tabla 5 se hallan descritos los ítems, las medias, variancias y cargas factoriales

\begin{tabular}{|c|c|c|c|}
\hline \multicolumn{4}{|c|}{ Tabla 5. Ítems del Big Eight +12} \\
\hline & Media & Variancia & $\begin{array}{l}\text { Peso factorial } \\
\text { (Factor) }\end{array}$ \\
\hline $\mathrm{D}_{1}$ & 3.88 & 2.37 & .50 \\
\hline D2 & 3.46 & 3.54 & .51 \\
\hline D3 & 3.12 & 0.51 & .30 \\
\hline $\mathrm{D}_{4}$ & 3.22 & 2.37 & .41 \\
\hline $\mathrm{M}_{1}$ & 4.24 & 1.21 & .46 \\
\hline $\mathrm{M} 2$ & 3.93 & 1.93 & .28 \\
\hline M3 & 4.06 & 1.48 & .39 \\
\hline$M_{4}$ & 3.19 & 2.61 & .46 \\
\hline M5 & 4.49 & 1.40 & .39 \\
\hline $\mathrm{F}_{1}$ & 3.00 & 2.32 & .34 \\
\hline $\mathrm{F}_{2}$ & 3.94 & 3.77 & .42 \\
\hline F3 & 3.95 & 2.24 & .37 \\
\hline $\mathrm{F}_{4}$ & 4.06 & 1.29 & .25 \\
\hline F5 & 3.57 & 2.66 & .42 \\
\hline F7 & 3.36 & 2.05 & .35 \\
\hline $\mathrm{T}_{1}$ & 3.54 & 1.00 & .39 \\
\hline $\mathrm{T}_{2}$ & 3.68 & 1.63 & .36 \\
\hline
\end{tabular}

[Josefina SALA-ROCA, Mercè JARIOT-GARCIA, Laura ARNAU-SABATÉS \& Eduardo DOVAL] SIPS - PEDAGOGÍA SOCIAL. REVISTA INTERUNIVERSITARIA [(2O21) 37, 39-53] TERCERA ÉPOCA

Copyright $\odot 2015$ SIPS. Licencia Creative Commons Attribution-Non Commercial (by-nc) Spain 3.0

\begin{tabular}{|c|c|c|c|}
\hline T3 & Media & Variancia & $\begin{array}{c}\text { Peso factorial } \\
\text { (Factor) }\end{array}$ \\
\hline T6 & 3.06 & 2.22 & .46 \\
\hline Del1 & 3.74 & 2.69 & .33 \\
\hline Del2 & 3.48 & 2.42 & .46 \\
\hline Del3 & 3.96 & 0.82 & .34 \\
\hline Del4 & 3.81 & 3.02 & .40 \\
\hline
\end{tabular}

\section{Discusión y conclusiones}

Si bien hay un gran consenso respecto al papel clave que juegan las competencias básicas o clave de empleabilidad en los procesos de inserción, mantenimiento y promoción laboral, las expectativas respecto a donde deben desarrollarse varían. Rosenberg et al., (2012) señalan que los estudiantes esperan desarrollarlas en el puesto laboral, mientras que los empleadores se quejan de que las universidades no las forman adecuadamente. Una parte considerable de los estudios sobre competencias clave o básicas de empleabilidad se han focalizado en la formación superior. No obstante, las competencias de empleabilidad básicas y transversales transferibles a un amplio espectro de puestos de trabajo empiezan a desarrollarse mucho antes; se aprenden en la infancia y adolescencia con el acompañamiento educativo de la familia y de los educadores y profesores, y se transfieren posteriormente al ámbito laboral. Estas competencias son muy relevantes para aquellos jóvenes que por su nivel formativo bajo pueden tener especiales dificultades en la inserción laboral, y que se encontraran en contextos de gran movilidad laboral cambiando frecuentemente de puesto de trabajo e incluso de sector económico. En la formación y orientación laboral de jóvenes procedentes de entornos desfavorecidos, resultan fundamentales modelos de competencias básicas de empleabilidad que permitan el acceso y mantenimiento del puesto de trabajo como el propuesto por Arnau, et al. (2014). No obstante, este instrumento puede ser utilizado con todo el espectro de jóvenes, independientemente de si su entorno es normalizado o desfavorecido, si residen en una familia o en un centro residencial. Las competencias propuestas en este modelo tienen un amplio consenso, habiendo sido validadas tanto por expertos como por trabajadores y son transferibles a múltiples escenarios: laborales, escolares, sociales y familiares. Por ello, 
consideramos que su evaluación es esencial en edades tempranas.

La revisión de la literatura sobre la evaluación de competencias clave para la empleabilidad demuestra la existencia de un vacío en el desarrollo de instrumentos psicométricos para evaluar competencias básicas de empleabilidad en adolescentes y jóvenes, como el desarrollado en este artículo; por lo que se han venido utilizando instrumentos que no han sido comprobados psicométricamente (Gibb, 2014; Cabrera et al., 2015; Clarke, 2016). El test situacional DCBE cubre este vacío. Es un test dirigido a jóvenes entre 12 y 18 años diseñado para evaluar estas competencias a partir de posibles situaciones representativas para este colectivo. Los autores han optado por desarrollar un instrumento copyleft con el fin de facilitar su utilización por parte de los profesionales de la Pedagogía Social, que frecuentemente se encuentran trabajando en entidades con fuertes limitaciones presupuestarias. El formato del instrumento como test situacional aporta una gran validez ecológica al permitir contextualizar las respuestas a situaciones problema de la vida real, y no meramente en valoraciones o percepciones de uno mismo (Lievens, et al., 2008). Esta proximidad conceptual con situaciones relevantes se sustenta en la selección de situaciones-problema de la vida cotidiana a los que se puede enfrentar cualquier adolescente, cuya representatividad y relevancia han sido validadas a partir del juicio de 102 jóvenes pertenecientes a la población diana.

Como se ha señalado, el modelo teórico en el que se fundamenta el test, había sido validado por un grupo de expertos en el ámbito de la inserción laboral con un alto nivel de acuerdo, lo que es destacable dada la gran variedad de clasificaciones de competencias de empleabilidad (Cinque, 2016) y, además, como indican Gulikers et al., (2010) porque su definición es compleja puesto que pueden adoptar distintas formas en función del contexto. Asimismo, previamente al desarrollo del test se validó la necesidad de dichas competencias para el acceso, mantenimiento y promoción en un amplio espectro de puestos de trabajo, cualificados y no cualificados, a partir de la experiencia de trabajadores de los cuatro sectores económicos (Jariot et al. 2021).

Después de diseñar historias y situaciones del test, un equipo de especialistas académicos y de selección de personal validó el contenido del test, alcanzando también buenos niveles de acuerdo. Respecto a la estructura del test, si bien se crearon las diferentes situaciones del test considerando las 8 competencias del modelo teórico, del análisis factorial exploratorio emergió una estructura unifactorial de 23 ítems, con pesos considerables en 20 de ellos. El hecho de que el análisis no permitiera corroborar una estructura de 8 escalas podría deberse al hecho de que en la resolución de las diferentes situaciones planteadas en el test situacional convergen diversas competencias y el test tiene un número de ítems inferior al que se recomienda desde el punto de vista psicométrico en el análisis de una estructura de 8 escalas. Por otra parte, la naturaleza de las puntuaciones del DCBE también supone una dificultad añadida ya que no tenían distancias uniformes. Las 5 opciones de respuesta no tenían una gradación y tampoco los expertos asignaban un valor gradual como sucede en los instrumentos en que se solicita a los participantes valorar en que grado o frecuencia se realiza una conducta o se experimenta alguna cosa. Este es un aspecto que dificulta la obtención de buenos resultados en el análisis factorial (Frías-Navarro \& Pascual Soler, 2012) en este instrumento. Pero precisamente el confrontar las competencias a contextos y situaciones específicas aporta mayor validez ecológica a la prueba (Kvavilashvili \& Ellis, 2004), aportando también una mayor validez incremental (Webster, et al., 2O2O). No obstante, la solución unifactorial tenía unos buenos valores de ajuste y de consistencia interna.

También cabe destacar el formato de la prueba en historias que conlleva un mayor interés por parte de los alumnos facilitando la concentración en la prueba sin el cansancio que suelen comportar muchas pruebas diagnósticas, que tanto profesores como alumnos encontraron el test útil, y que estos últimos comentaron que se veían representados en las puntuaciones.

El estudio también tuvo algunas limitaciones que en el futuro deben abordarse. Por una parte, la administración fue realizada por los profesores, que fueron instruidos para ello. No obstante, si hubiera sido factible administrarlos por los investigadores las condiciones de aplicación habrían sido más homogéneas. Por otra, es necesario incluir algún ítem de control de deseabilidad social que pueda permitir compensar este efecto. El desarrollo de un test, es un proceso largo y requiere de muchos estudios. Hasta el momento se ha validado el modelo en el que se sustenta el test, se ha validado el contenido del test por expertos, se ha estudiado su naturaleza estructural y la fiabilidad de la solución unidimensional.

En conclusión, el test DCBE es un instrumento psicoeducativo para adolescentes y jóvenes que permite a los profesionales de la Pedagogía Social y a otros profesionales educativos valorar el nivel de desarrollo de las competencias básicas de empleabilidad que se requieren para 
acceder, mantener y promocionar en cualquier trabajo. A estas competencias básicas se sumarán las competencias profesionales específicas de la profesión y del puesto de trabajo, pero que serán insuficientes sin las competencias básicas de empleabilidad. El test posibilita iniciar la preparación para la empleabilidad en etapas tempranas previas a la formación postobligatoria al mismo tiempo que incrementa el potencial éxito de la formación profesionalizadora, y la inserción laboral posterior. Este estudio ha aportado evidencias de la validez de contenido evaluada por jueces y de validez de constructo, así como de la buena aceptación por su atractivo y utilidad tanto entre los alumnos como entre los profesores. Su carácter copyleft permitirá que muchos profesionales de la Pedagogía Social puedan utilizarlo en los diferentes contextos de acción socioeducativa con jóvenes. El test se halla disponible en https://ddd. uab.cat/record/235416.

En futuros estudios debería poder confirmarse la estructura del test, aplicando, por ejemplo, un análisis factorial confirmatorio, explorarse la estabilidad temporal de las puntuaciones del test y hallarse otras evidencias de validez criterial que contribuyan a reforzar las características psicométricas del instrumento.

\section{Agradecimientos}

Este estudio se ha realizado con la financiación del Ministerio de Economía y Competitividad, y cofinanciado por FEDER (EDU2013-43326-R y EDU2016-77284-R). Queremos asimismo agradecer a los participantes, los centros educativos y el ayuntamiento de Mollet su colaboración en el estudio.

\section{Referencias bibliográficas}

Abas, M. C., \& Imam, O. A. (2016). Graduates' Competence on Employability Skills and Job Performance. International Journal of Evaluation and Research in Education, 5(2), 119-125.

Alonso Bello, E.; Santana Vega, L.E., \& Feliciano García, L. (2018). Trayectorias de empleabilidad de los jóvenes que abandonan el sistema de protección. Educación Siglo XXI, 36(3), 485-504. https://doi.org/10.6018/j/350101

Arnau-Sabatés, L., Marzo, M. T., Jariot, M., \& Sala-Roca, J. (2014). Learning basic employability competence: a challenge for the active labour insertion of adolescents in residential care in their transition to adulthood. European Journal of Social Work, 17(2), 252-265. https://doi.org/10.1080/13691457.2013.802227

Blalock, L. B., Strieter, L., \& Hughes, L. (2006). The SCANS Skills and Competencies Checklist: An assessment tool for youth work readiness programs. Journal of Youth Development, 17), 89-99.

Breen, R. (2005). Explaining cross-national variation in youth unemployment market and institutional factors. European sociological review, 21(2), 125-134. https://doi.org/10.1093/esr/jcioo8

Bridgstock, R. (2009). The graduate attributes we've overlooked: Enhancing graduate employability through career management skills. Higher Education Research \& Development, 28(1), 31-44. https://doi.org/10.1080/07294360802444347

Cabrera, Lanzo, N., López López, M.C., \& Portillo Vidiella, M.C. (2015). La percepción de los empleadores acerca de las competencias de los graduados y su evaluación. Una aproximación a distintos sectores profesionales. En Londoño Orozco, Guillermo and Cano García, Elena: Formación y evaluación por competencias en educación superior. (185213). https://doi.org/10.19052/9789588844992

Cimatti, B. (2016). Definition, development, assessment of soft skills and their role for the quality of organizations and enterprises. International Journal for quality research, 10(1). https://doi.org.10.18421/IJQR10.01-05

Cinque, M. (2016). "Lost in translation”. Soft skills development in European countries. Tuning Journal for Higher Education, 3(2), 389-427. http://dx.doi.org/10.18543/tjhe-3(2)-2016pp389-427

Clarke, M. (2016). Addressing the soft skills crisis. Strategic HR Review, 15(3), 137-139. https://doi.org/10.1108/SHR-032016-0026

Crisp, R., \& Powell, R. (2016). Young people and UK labour market policy: A critique of 'employability' as a tool for understanding youth unemployment. Urban Studies, 54(8), 1784-1807. https://doi.org/10.1177/0042098016637567

Curtis, D., \& McKenzie, P. (2001). Employability skills for Australian industry: Literature review and framework development. Report to: Business Council of Australia; Australian Chamber of Commerce and Industry. Science and Training, Canberra. http://hdl.voced.edu.au/10707/40939.

Dreger, C., López-Bazo, E., Ramos, R., Royuela, V., \& Suriñach, J. (2015). Wage and income inequality in the European Union. Policy Department. Retrieved from http://ec.europa.eu/eurostat/cros/system/files/O5-2014-wage_and_income_inequality_in_the_eu_o.pdf

Eurostat (2020). Unemployement statistics. http://ec.europa.eu/eurostat/statistics-explained/index.php/Unemployment_statistics 
Fleiss, J. L. (1981) Statistical methods for rates and proportions. 2nd ed. (New York: John Wiley) pp. 38-46.

Frías-Navarro, D., \& Pascual Soler, M. (2012). Exploratory factor Analysis (EFA) in consumer behavior and marketing research. Suma Psicológica, 19(1), 47-58. Disponible en: http://www.scielo.org.co/scielo.php?script=sci_arttext\&pi$d=$ So121-43812012000100004

Gibb, S. (2014). Soft skills assessment: theory development and the research agenda. International Journal of Lifelong Education, 33(4), 455-471. https://doi.org/10.1080/02601370.2013.867546

Griffiths, D. A., Inman, M., Rojas, H., \& Williams, K. (2018). Transitioning student identity and sense of place: future possibilities for assessment and development of student employability skills. Studies in Higher Education, 43(5), 891-913.

Gulikers, J. T., Baartman, L., \& Biemans, H. J.(2010). Facilitating evaluations of innovative, competence-based assessments: Creating understanding and involving multiple stakeholders. Evaluation and Program Planning, 33(2), 12O127. https://doi.org/10.1016/j.evalprogplan.2009.07.002

Jariot, M.; Sala, J., Arnau, L. \& Marzo, M. (2021). Validación del modelo teórico del Test Situacional de Desarrollo de las Competencias Básicas de Empleabilidad: la perspectiva de los trabajadores. Revista de Pedagogía Social, 37, 1-16.

Kvavilashvili, L., \& Ellis, J. (2004). Ecological validity and the real-life/laboratory controversy in memory research: A critical and historical review. History \& Philosophy of Psychology, 6, 59-80. Disponible en: https://uhra.herts.ac.uk/ bitstream/handle/2299/1971/103125.pdf?

Lievens, F., Peeters, H., \& Schollaert, E. (2008). Situational judgment tests: A review of recent research. Personnel Review, 37(4), 426-441. https://doi.org/10.1108/00483480810877598

Lo Presti, A. (2009). Snakes and ladders: Stressing the role of meta-competencies for post-modern careers. International Journal for Educational and Vocational Guidance, 9(2), 125-134. https://doi.org/10.1007/s10775-009-9157-0

Lo Presti, A. L., \& Pluviano, S. (2016). Looking for a route in turbulent waters Employability as a compass for career success. Organizational Psychology Review, 6(2), 192-211. https://doi.org/10.1177/2041386615589398

Lotito, F. (2015). Test Psicológicos y Entrevistas: Usos y Aplicaciones Claves En El Proceso De Selección E Integración De Personas a Las Empresas (Psychological Tests and Interviews: Key Uses and Applications in the Selection and Integration of Companies). RAN-Revista Academia \& Negocios, 1(2).

Lorenzo-Seva, U., \& Ferrando, P. (2007). FACTOR: A computer program to fit the exploratory factor analysis model. University Rovira y Virgili. Disponible en: https://link.springer.com/content/pdf/10.3758/BFo3192753.pdf

Lorenzo-Seva, U., \& Ferrando, P. (2011). Manual of the program FACTOR v. 8.02. Disponible en: https://psico.fcep.urv.es/ utilitats/factor/Manual-of-the-FactorProgram.pdf

MacCann, C., \& Roberts, R. D. (2008). New paradigms for assesing emotional intelligence: theory and data. Emotion, 8(4), 540-551. https://doi.org/10.1037/a0012746

McQuaid, R. W. (2006). Job search success and employability in local labor markets. The Annals of Regional Science, 4O(2), 407-421. https://doi.org/10.1007/s00168-006-0065-7

McQuaid, R. W., \& Lindsay, C. (2005). The concept of employability. Urban Studies, 42(2), 197-219. https://doi.org/10.1007/ s00168-006-0065-7

Moreno Mínguez, A. (2015). La empleabilidad de los jóvenes en España. Explicando el elevado desempleo juvenil durante la recesión económica. Revista Internacional de Investigación en Ciencias Sociales, 11(3), 3-20. https://doi. org/10.18004/riics.2015.julio.3-20

Muhamad, S. (2012). Graduate employability and transferable skills: A review. Advances in Natural and Applied Sciences, 6(6), 882-885. Disponible en: https://www.researchgate.net/profile/Suriyani_Muhamad/publication/287021703. Graduate_employability_and_transferable_skills_A_review/links/5da8703492851caalbabd804/Graduate-employability-and-transferable-skills-A-review.pdf

Nickson, D., Warhurst, C., Commander, J., Hurrell, S. A., \& Cullen, A. M. (2012). Soft skills and employability: Evidence from UK retail. Economic and Industrial Democracy, 33(1), 65-84. https://doi.org/10.1177/0143831x11427589

Nisha, S. M., \& Rajasekaran, V. (2018). Employability skills: A review. IUP Journal of Soft Skills, 12(1), 29-37. Disponible en. $\quad$ http://search.proquest.com/openview/1f9f5ad8e5798a6b992e4c1bee830cbb/1?pq-origsite=gscholar\&cbl=2029989\&casa_token=OGFchuXN-2gAAAAA:pkjjLEAu-RDiCNJioXkoDJTVjnjZqoKmLE4gqWIheZebJKGfq. YJw8j4lZkuzxps_j6kIMXLrJ4

Olea, M. A. (2001). El trabajo como bien escaso. Revista del Ministerio de Trabajo e Inmigración, 33, 17-32.

Olmos-Rueda, P., \& Mas-Torelló, O. (2017). Perspectiva de tutores y de empresas sobre el desarrollo de las competencias básicas de empleabilidad en el marco de los programas de formación profesional básica. Educar, 53(2), $261-284$. DOI: https://doi.org/10.5565/rev/educar.870

Organización Internacional del Trabajo (2014). Competencias para el empleo. Mejorar la empleabilidad de los jóvenes: La importancia de las competencias clave. Recuperado en http://www.ilo.org/skills/pubs/WCMS_371815/lang--es/ index.htm 
Riebe, L., \& Jackson, D. (2014). The use of rubrics in benchmarking and assessing employability skills. Journal of Management Education, 38(3), 319-344.

Rosenberg, S., Heimler, R., \& Morote, E. S. (2012). Basic employability skills: a triangular design approach. Education+ Training. https://doi.org/10.1108/00400911211198869

Rothwell, A., Herbert, I., \& Rothwell, F. (2008). Self-perceived employability: Construction and initial validation of a scale for university students. Journal of vocational behavior,73(1), 1-12.

Sala, J., Arnau, L., Jariot, M., Marzo, T., Urrea, A. \& Ortega, D. (2020). Test Situacional de Desarrollo de Competencias Básicas de Empleabilidad (DCBE). Versión revisada de J. Sala-Roca, L. Arnau, M. Jariot, T. Marzo. The Big Eight Test. DDD. https://ddd.uab.cat/record/235416

Sorrel, M. A., Olea, J., Abad, F. J., de la Torre, J., Aguado, D., \& Lievens, F. (2016). Validity and Reliability of Situational Judgement Test Scores A New Approach Based on Cognitive Diagnosis Models. Organizational Research Methods, 19(3), 506-532. https://doi.org/10.1177/1094428116630065

Webster, E. S., Paton, L. W., ES Crampton, P., \& Tiffin, P. A. (2020). Situational judgement test validity for selection: a systematic review and meta-analysis. Medical Education. https://doi.org/10.1111/medu.14201

\section{CÓMO CITAR ESTE ARTÍ́CULO}

Sala-Roca, J., Jariot-Garcia, M., Arnau-Sabatés, L., \& Doval, E. (2020). Test situacional desarrollo de las competencias básicas de empleabilidad: validación de un instrumento psicoeducativo para la intervención socioeducativa. Pedagogía Social. Revista Interuniversitaria, 37, 39-53. DOI: 10.7179/PSRI_2021.02

\section{DIRECCIÓN DE LOS AUTORES}

JOSEFINA SALA-ROCA. E-mail: Fina.Sala@uab.cat

MERCÈ JARIOT-GARCIA. E-mail:

LAURA ARNAU-SABATÉS. E-mail: laura.arnau@uab.cat

EDUARDO DOVAL DIÉGUEZ. E-mail: Eduardo.doval@uab.cat

\section{PERFIL ACADÉMICO}

JOSEFINA SALA ROCA. Profesora del departamento de Teories de l'Educació y Pedagogia Social de la Universidad Autónoma de Barcelona. Coordina el grupo de Investigación de Infancia y Adolescencia en Riesgo Social (IARS). El grupo IARS en colaboración con FEPA, FEDAIA y DGAIA ha desarrollado diversos estudios sobre los factores que inciden en el bienestar y desarrollo positivo de los jóvenes tutelados, así como de las dificultades y apoyos a su transición a la vida independiente. Las investigaciones desarrolladas se han publicado en diversos artículos e informes.

https://orcid.org/0000-0001-9829-5131 
MERCĖ JARIOT-GARCIA. Profesora del departamento de Pedagogia Aplicada de la Universidad Autónoma de Barcelona. Forma parte del grupo de Investigación de Infancia y Adolescencia en Riesgo Social (IARS). Sus líneas de investigación se relacionan con la transición de los jóvenes ex tutelados a la vida adulta, la inserción socio laboral, el cambio de actitudes y la mejora y desarrollo de las competencias de empleabilidad.

https://orcid.org/oooo-0002-5061-2940

LAURA ARNAU-SABATÉS. Profesora del departamento de Teorías de la Educación y Pedagogía Social de la Universidad Autónoma de Barcelona. Forma parte del grupo de Investigación de Infancia y Adolescencia en Riesgo Social (IARS). Sus líneas de investigación se relacionan con la protección a la infancia tutelada, la transición de los jóvenes ex tutelados a la vida adulta, la inserción socio laboral y la mejora y desarrollo de las competencias de empleabilidad.

https://orcid.org/0000-0003-3359-4071

EDUARDO DOVAL DIÉGUEZ. Profesor del departamento de Psicobiología y de Metodología de las Ciencias de la Salud de la Facultad de Psicología de la Universidad Autónoma de Barcelona. Miembro del grupo de investigación en estrés y salud (GIES). Ha desarrollado diversos estudios relacionados con la adaptación de instrumentos de medida y con la evaluación de la calidad métrica de los instrumentos de medida, en especial con la evaluación de patrones atípicos de respuesta. Sus investigaciones se han publicado en diversos artículos, libros e informes técnicos. https://orcid.org/o000-0001-8416-160X 
